\title{
TUMORES DEL OVARIO EN LA INFANCIA
}

\author{
Drs. JULIO ESPINOZA y RENE ARTIGAS \\ Instituto de Anatomia Patológía y Servicio de Cirugía Infantil. \\ Hospital "Manuel Arriarán". Santiago.
}

La presencia de tumores ováricos en la infancia, ha sido señalada como un hecho raro en la literatura nacional y foránea. Numerosos autores están acordes en considerar para el ovario una incidencia del $1 \%$ de todos los tumores encontrados en niños de ambos sexos hasta la edad de 15 años. También existe amplio concenso en atribuir al ovario, desde edades muy tempranas, una capacidad enorme para desarrollar tumores que figuran entre los más grandes del cuerpo, tumores que poseen una estructura que va desde las más simples hasta las más complejas y con una sintomatología, evolución $y$ pronóstico muy peculiares. Tal vez conviene recordar aquí la no despreciable incidencia con que estos neoplasmas evolucionan hacia la malignidad y la alta frecuencia con que acontece en ellos el dramático accidente de la torsión del pedículo, hecho este último que no rara vez es el primer síntoma aparente de la afección blastomatosa y que pone en grave peligro la vida de la enfermita. Resultan así, tanto desde el punto de vista morfológico como desde el punto de vista clínico, padecimientos que suscitan marcado interés y que invitan a sucesivos estudios y revisiones.

Entre nosotros, los tumores ováricos de las niñas han sido dados a conocer como casos aislados por diversos autores hasta 1949, en que realiza su Tesis de Médico el Dr. Ariztía Montt el cual recopila 17 casos de diferentes Hospitales de Santiago y Valparaíso y en amena y documentada exposición los analiza en sus aspectos clínico y anátomo-patológico. Del Hospital de Niños "Manuel Arriarán", única fuente de nuestra casuística, refiere 5 casos, que también incluímos nosotros.

Nuestro material está compuesto por 21 casos, 5 de los cuales corresponden a necropsias (Cuadro 1).

En nuestra exposición sólo nos referimos a aqueilos tumores que tienen representación en esta casuística, para lo cual los agrupamos según el diagnóstico histopatológico. En cada grupo exponemos pri-
CUADRO NO 1

RESUMEN DE NUESTRO MATERIAL

(2l castos)

\begin{tabular}{|c|c|c|}
\hline Tipo de tumor & N8 cmon & Porcentaje \\
\hline 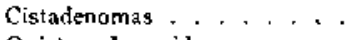 & 6 & 28,5 \\
\hline $\begin{array}{l}\text { Quistes dermoides } \ldots \ldots . \\
\text { Teratonias benignos } \ldots \ldots \ldots \\
\text { Teratomas malignos } \ldots \ldots . .\end{array}$ & $\begin{array}{l}5 \\
4 \\
2\end{array}$ & 52,0 \\
\hline Disgerminomas . . . . . & 2 & 9,5 \\
\hline Carcinomas sólidos , . , , & 2 & 9,5 \\
\hline
\end{tabular}

mero suscintamente las nociones etio-patogénicas clínicas y anátomo-patológicas elementales, anotando también la experiencia de otros autores $y$ en seguida relatamos nuestros casos y los analizamos en un breve comentario parcial. Hecho esto en cada grupo, finalizamos el trabajo con un Comentario General y Conclusiones.

\section{Cistadenomas}

Comprenden dos tipos: el seroso y el pseudomucoso. Aunque difieren en diversos aspectos, tales como en su etiología e histopatología, tienen de común el ser conocidos desde los primeros tiempos de la cirugía - se supone que la primera operación sobre los ovarios de que hay constancia en la historia de la medicina, practicada por Efraín Mc. Dowel, fué la extirpación de un cistadenoma mucoso, en 1809 - su frecuencia es la más alta dentro de los neoplasmas ováricos y a ambos corresponde una estructura generalmente benigna.

La frecuencia de los cistadenomas en la infancia, ha sido considerada precaria por la mayoría de los autores. Costin y Kennedy dan un valor del $11 \%$ y Huber del 14\%. Sin embargo, Dargeon hace subir esta incidencia al $32 \%$. Por lo demás, se encuentran en la literatura casos aislados y pequeñas casuísticas de este tipo de quistes en recién nacidos (Smith) y prematuros (Graves y colaboradores), algu- 
nos de los cuaies dieron lugar por su tamaño a distocias y en otros a ruptura del quiste en el momento del parto con muerte del feto.

En nuestra serie de 21 casos, 6 correspondieron a citadenomas, to cual significa una frecuencia de $28,5 \%$. Nuestro caso más joven fué el de una niña de 1 mes de edad y 4 de los 6 casos son menores de 1 año (Cuadro $\mathrm{N}^{9} 2$ ). De las dos niñas con 12 años, sólo una menstruaba (caso 4), desde hacía 2 años, lo que fué considerado como pubertad precoz. El tumor y dolor abdominal fueron los síntomas predominantes, ya concomitantes o aislados en 4 casos ( $\mathrm{N}^{\circ}$ 3, 4, 5 y 6 ). En dos casos ( $\mathrm{N}^{\circ} 1$ y 2), no hubo sintomatología alguna que acusara la presencia del quiste, pero su tamaño, en ambos, era muy reducido $(0.8$ $\mathrm{cm}$. y $1,5 \mathrm{~cm}$. respectivamente) y sólo fueron hallazgos de autopsia. Sin embargo, en el $4 \mathrm{y}$ pese a lo pequeño del tumor (1 $\mathrm{cm}$. de diámetro solamente) hubo manifestaciones dolorosas abdominales durante 5 días que hicieron suponer una apendicitis sub-aguda y fué operada con tal diagnóstico. El dolor en este caso no pudo explicarse por torsión importante del quiste, pues en el examen histológico no se descubrió ningún signo evidente de esa complicación. En el último caso ( $N^{\circ}$ 6) no se palpó el tumor a través de la pared abdominal, quizás por el exquisito dolor que presentaba en la fosa ilíaca derecha acompañado de defensa muscular, síntomas que, por lo demás, hicieron pensar en una apendicitis aguda, diagnóstico con el cual se operó y que tal vez indujo a omitir el tacto rectal. En la intervención se comprobó la existencia de un tumor quístico torcido. En otro caso ( $\mathbf{N}^{\circ} \mathbf{3}$ ), a la enfermita, afecta de grave distrofia, se le palpó una masa abdominal del hipogastrio, del tamaño de una naranja, a la cual se puncionó dando salida a 20 cc. de lí- quido café-obscuro cuyo análisis reveló abundante albuminosis (40 grs.) y glóbulos rojos en gran parte destruídos. Se pensó por ésto, en un hematoma en reabsorción de origen no precisado. La evolución rápidamente desfavorable de la enferma, que falleció a los 25 días de hospitalizada, no hizo posible otras investigaciones en ese detalle de su grave afección nutritiva y en autopsia se descubrió un cistadenoma seroso con signos frescos de infarto hemorrágico, complicación que lógicamente transcurrió en forma inaparente ya que debió producirse en el o los días que precedieron a su fallecimiento. En la niña de un mes (caso 5) el síntoma principal fué la constipación, dato que suministrado por la madre lo hace remontar hasta el nacimiento. Muy probablemente esta niña nació con la neoformación que comprimía el recto y daba lugar al mencionado síntoma, que desapareció después de la operación.

Resulta importante comprobar lo frecuente de la torsión del tumor quístico, 3 veces en 6 casos ( $50 \%$ ) y correspondiendo este accidente a los cistadenomas de mayor tamaño (sobre $5 \mathrm{~cm}$. de diámetro), lo cual sugiere alguna relación de causa a efecto en la producción del fenómeno que comentamos.

El tratamiento de los casos operados (4, 5 y 6) consistió en salpingooforectomía unilateral en los dos primeros y ooforectomía unilateral en el otro. Las tres enfermitas convalecieron sin inconvenientes.

a) Cistadenoma seroso. - Su histogénesis, como la de la mayoría de los tumores ováricos, no está totalmente esclarecida. La opinión más generalizada es que tiene su origen en el epitelio germinal del ovario. Generalmente es unilateral y unilocular. Dockerty lo ha observado en ambos ovarios en el $30 \%$ de sus casos. El tamaño es muy variable, pudiendo alcan-

CUADRO No 2

C I S T D ENO MA S

(6) crsos: 28,5 \%

\begin{tabular}{|c|c|c|c|c|c|c|c|}
\hline $\begin{array}{l}\text { No de } \\
\text { Orden }\end{array}$ & $\begin{array}{l}\text { Necmopaiz } \\
\text { o Biogsia }\end{array}$ & & Edad & Lado & Torsion & Tஃmąก๊o & $\begin{array}{l}\text { Diagnostico } \\
\text { anat,-pat. }\end{array}$ \\
\hline $\begin{array}{l}1 \\
2 \\
3 \\
4 \\
5 \\
6\end{array}$ & $\begin{array}{rr}\text { N. } & 68 / 46 \\
\mathbf{N} . & 402 / 46 \\
\text { N. } & 419 / 51 \\
\text { B. } & 73 / 52 \\
\text { B. } & 229 / 54 \\
\text { B. } & 161 / 48\end{array}$ & $\begin{array}{l}4 \\
11 / 2 \\
9 \\
12 \\
1 \\
12\end{array}$ & $\begin{array}{l}\text { meses } \\
\text { meses } \\
\text { meses } \\
\text { años } \\
\text { mes } \\
\text { años }\end{array}$ & $\begin{array}{l}\text { Izquierdo } \\
\text { Izquierdo } \\
\text { Izquier do } \\
\text { Derecho } \\
\text { Izquierdo } \\
\text { Izquierdo }\end{array}$ & $\begin{array}{l}\text { Ná } \\
\text { Nó } \\
\text { Sij } \\
\text { No } \\
\text { Sij } \\
\text { Sij }\end{array}$ & $\begin{array}{r}0,8 \mathrm{cms} . \\
1,5 \mathrm{cms} . \\
5 \times 3 \mathrm{cms} . \\
1 \mathrm{~cm}, \\
6 \times 4 \mathrm{cms} . \\
10 \times 5 \mathrm{cms} .\end{array}$ & $\begin{array}{l}\text { C.S. } \\
\text { C.S. } \\
\text { C.S. } \\
\text { C.S. } \\
\text { C.S. } \\
\text { C.M. }\end{array}$ \\
\hline
\end{tabular}

Abreviaciones: C.S. = Cistadenoma Seroso. C.M. = Cistadenoma Mucoso. 
zar volúmenes enormes si se le deja evolucionar espontáneamente. Su contenido está formado por líquido fluído o espeso, claro o teñido con sangre, hasta el color café. Microscópicamente está constituído por pared fibrosa, en general delgada, pero resistente, que incluye vasos sanguineos y linfáticos y glándulas limitadas por epitelio cúbico. El revestimiento epitelial del quiste es también de tipo cúbico - aplanado, según el grado de tensión interior que puede hasta hacerlo desaparecer en algunas zonas. Según Wharton, puede observarse un cistadenoma mixto (seroso-pseudomucoso), encontrándose entonces porciones de ambos tipos histológieos.

En nuestra serie, los 5 casos de cistadenomas serosos, eran uniloculares y unilaterales, 4 del ovario izquierdo y sólo uno del derecho. En todos ellos la pared era delgada, de pocos milímetros de espesor, de color blanquecino, que mostraba focos hemorrágicos en los que habían sufrido torsión (casos 3 y 5). Su contenido estaba formado por líquido blanquecino transparente, teñido de rojo en los mismos casos señalados. EI epitelio de recubrimiento interno era aplanado $y$ discontinuo en tres de ellos (casos 1, 2 y 5), de células cúbicas $\mathrm{y}$ columnares dispuestas en una o dos capas en el caso 4. Este último mostraba en escasos puntos leves solevantamientos papilares revestidos por idénticos elementos celulares a los del resto de su pared. En el espesor de la pared de dos casos $\left(\begin{array}{lll}4 & \text { y } & 5\end{array}\right)$ eran claramente visibles pequeños acúmulos de glándulas limitadas por epitelio cúbico dispuestas en una sola capa. En los casos 3 y 5 se apreciaban además, focos hemorrágicos y necróticos intramulares; en el último aparecian numerosos y pequeños depósitos cálcicos diseminados en la pared fibrosa.

b) Cistadenoma pseudomucoso. - Su histogénesis también es incierta. Algunos los bacen derivar del epitelio entodérmico en un teratoma (Novak y otros). Schiller, Barzilai. Ewing, etc. le suponen un origen mulleriano, nacido de restos del cuerpo de Müller o de epitelio germinal del ovario que sufre proceso de metaplasia. Generalmente es unilateral y multilocular. El tamaño es moderado o grande, sin limites, ya que antiguamente se observaron casos de $40 \mathrm{y}$ más kilos de peso, so- brepasando en ocasiones al propio peso de la enferma. Su color es azul o grisáceo, según el color del líquido que contenga: La consistencia es firme, más en algunas zonas que en otras, dependiendo del espesor de la pared y del proceso proliferativo tisular. El contenido está formado por líquido espeso, viscoso, que contiene pseudomucina. Microscópicamente, la pared es fibrosa, revestida en su superficie interna por células prismáticas ultas, dispuestas en una sola capa, de citoplasma transparente y núcleo basal bien teñido, entre las que se destacan algunas células caliciformes. En el espesor de la pared suelen observarse glándulas de igual estructura al epitelio descrito. Aunque es un tumor benigno, la ruptura y vaciamiento de su material gelatinoso en el peritoneo puede llegar a producir la muerte, por crecimiento gradual de esa substancia a partir de células tumorales que se implantan en aquella serosa: es el cuadro denominado "pseudomixoma del peritoneo", capaz de ser producido también por el apéndice. Para la mayoría de los investigadores, el pseudomixoma peritoneal es clínicamente maligno, aunque benigno desde el punto de vista histopatológico. Las proliferaciones papilares, se observan según Costin $y$ Kennedy en un $10 \%$ de estos tumores $\mathbf{y}$ su evolución maligna, de acuerdo con estos mismos autores, ocurriría en el $5 \%$ más o menos.

En nuestra serie sólo encontramos un caso de cistadenoma pseudomucoso ( $\mathrm{N}^{\circ}$ 6), niña que ingresó por cuadro abdominal agudo y en que se intervino con el diagnóstico de Apendicitis Aguda. Al abrir el peritoneo salió pequeña cantidad de sangre líquida y se encontró un quiste torcido del ovario izquierdo, de color rojoobscuro y de consistencia renitente. Al corte, aparecía con múltiples cavidades de tamaño diferente, separadas por delgados tabiques, también rojo-obscuros y de aspecto hemorrágico. Su contenido estaba formado por abundante líquido rosado, mucilaginoso. El examen microscópico reveló pared fibrosa revestida por epitelio cilíndrico muy claro y núcleo basal intensamente teñido. En numerosas y extensas zonas se encontraron infiltraciones hemorrágicas y necrosis de los elementos celulares. La trompa estaba totalmente afectada por el infarto hemorrágico. 


\section{Teratomas.}

Se presentan de preferencia en personas jóvenes. En antigua estadística de Seyfarth (1916), figuraban 171 casos en niñas menores de 15 años. Según Ewing, Barzilai y Lisa y col., los teratomas sólidos son más frecuentes en los años premenstruales, mientras el quiste dermoide aumenta su incidencia entre el segundo y tercer decenio de la vida. Para Kroemer, el quiste "dermoide constituye el $10 \%$ de todos los tumores del ovario, encontrándose el teratoma sólido en una proporción mucho menor. En la serie de Costin y Kennedy, de 22 tumores, 10 eran teratomas $(45,5 \%)$ que incluían 7 quistes dermoides y 3 teratomas sólidos. Como un caso raro de precocidad en su presentación, encontramos la publicación de $\mathrm{Ga}$ rret, que relata el hallazgo de un teratoma quístico benigno en una niña de 2 meses, el cual fué extirpado 7 meses después.

Nuestra serie consta de 11 teratomas $(52 \%)$, de los cuales 5 eran quistes dermoides, 4 teratomas quísticos benignos y 2 teratomas sólidos malignos (Cuadro No 3).

Respecto de su edad, la menor era de 3 años, tres entre 8 y 9 años y siete entre 10 y 11 años.

Los síntomas y signos principales fueron: dolor $\mathrm{y}$ tumor. Prácticamente en todos los casos (a excepción del No 7 que fué hallado en autopsia), las enfermitas acusaban dolor medianamente agudo con exacerbaciones periódicas, durante 2 a 5 días. En 7 casos se palpó el tumor por vía abdominal, en 2 de los cuales se corroboró por el tacto rectal. En las 3 restantes, la palpación abdominal no descubrió el tumor, ni tampoco el tacto rectal practicado en una de éstas. Estos 3 casos (8, 11 y 17) presentaban intenso dolor $y$ defensa muscular abdominales por lo que tal vez la palpación fué infructuosa y el diagnóstico pre-operatorio fué de apendicitis aguda. En otros dos casos ( 13 y 14) en que también la defensa abdominal impedia el examen palpatorio y ya decidida la intervención, se anestesió la enferma y entonces la palpación reveló la masa tumoral en el hemiabdomen inferior. En 5 enfermitas se agregó vómitos de uno a tres días de duración, comprobándose en ellas acentuada defensa abdominal y coincidiendo estos signos con torsión del tumor ovárico. En 4 enfermitas, la historia acusaba antiguos padecimientos dolorosos abdominales de poca monta y transitorios, que variaban de 1 a 2 meses en tres de ellas (12, 15 y 16) y hasta un año antes en la cuarta (caso 8). En dos casos. ( $\mathrm{N}^{\circ} 9$ y 14) se hizo radioscopía intestinal, pero no se visualizaron las calcificaciones del quiste dermoide. Este recurso, según Costin y Kennedy, permite reconocer el $41 \%$ de tales tumores. El diagnóstico pre-operatorio de estos 11 casos de Teratomas fué de quiste del ovario en 5 ( 2 veces con interrogativo), de tumor abdominal en 2 casos y de apendicitis aguda en 3 . En un caso ( $N$ \% ) el tumor fué hallazgo de autopsia, pues la enfermita, afecta de grave cuadro abdominal agudo por rotura traumática del intestino, sólo permaneció con vida breves horas y su quiste dermoide pasó totalmente inadvertido.

Respecto de las complicaciones de los Teratomas, todos los autores están de acuerdo en que la torsión del pedículo es la más frecuente, observándose una mayor incidencia de esta complicación en los quis-

CUADRO N'Q 3

T E R A T O M A S

(11 casus: $53 \%$ )

\begin{tabular}{|c|c|c|c|c|c|c|c|}
\hline $\begin{array}{l}\text { Orden } \\
\text { No de }\end{array}$ & $\begin{array}{l}\text { Necropsia } \\
\text { o Biopsia }\end{array}$ & Edad & Lado & Torsi6e & Tamaña & Urgencia & $\begin{array}{l}\text { Diagnostico } \\
\text { anat.-pat. }\end{array}$ \\
\hline $\begin{array}{r}7 \\
8 \\
9 \\
19 \\
11 \\
12 \\
13 \\
14 \\
15 \\
16 \\
17\end{array}$ & $\begin{array}{lr}\text { N. } & 85 / 44 \\
\text { B. } & 8 / 45 \\
\text { B. } & 5 / n \\
\text { B. } & 40 / 48 \\
\text { B. } & 15 / 49 \\
\text { B. } & 197 / 52 \\
\text { B. } & 26 / 55 \\
\text { B. } & 171 / 55 \\
\text { B. } & 85 / 56 \\
\text { B. } & 20 / 53 \\
\text { B. } 197 / 55\end{array}$ & $\begin{aligned} 11 & \text { años } \\
10 & \text { años } \\
11 & \text { años } \\
10 & \text { años } \\
8 & \text { años } \\
10 & \text { años } \\
8 & \text { años } \\
11 & \text { años } \\
3 & \text { años } \\
10 & \text { años } \\
9 & \text { años }\end{aligned}$ & $\begin{array}{l}\text { Derechro } \\
\text { Derecho } \\
\text { Derecho } \\
\text { Derecho } \\
\text { Derecho } \\
\text { Detecho } \\
\text { Izquierdo } \\
\text { Jzquierdo } \\
\text { Izquietslo } \\
\text { Izquaerdo } \\
\text { Derecho }\end{array}$ & $\begin{array}{l}\text { Nín } \\
\text { Sí } \\
\text { Nió } \\
\text { Sii } \\
\text { Si } \\
\text { Si } \\
\text { Sí } \\
\text { Sí } \\
\text { Nó } \\
\text { Sí } \\
\text { Roto }\end{array}$ & $\begin{array}{r}1,5 \text { cms } \\
9 \times 5 \text { cms } \\
4 \text { cms. } \\
9 \times 6 \text { cuss. } \\
7 \times 5 \text { cms. } \\
14 \times 10 \text { cms. } \\
10 \times 8 \text { cms. } \\
99 \text { cms. } \\
9 \times 6 \text { cms. } \\
15 \mathrm{cms} . \\
10 \times 6 \text { cms. }\end{array}$ & $\begin{array}{l}\text { Nó } \\
\text { sí } \\
\text { Só } \\
\text { Sii } \\
\text { Si } \\
\text { Si } \\
\text { Si } \\
\text { Si } \\
\text { Si } \\
\text { Sí } \\
\text { Sí }\end{array}$ & $\begin{array}{l}\text { Q.D. } \\
\text { Q.D. } \\
\text { Q.N. } \\
\text { Q.D. } \\
\text { D.D. } \\
\text { T.B. } \\
\text { T.B. } \\
\text { T.B. } \\
\text { T.B. } \\
\text { T.M. } \\
\text { T.M. }\end{array}$ \\
\hline
\end{tabular}

Abreviaciones: Q.D. = Quiste Dermoile. T.B. = Teratoma Benigno. T.M. = Teratoma Maligno. 
tes dermoides (12\% según Marshall). Las consecuencias de la torsión varían según el grado de la obstrucción vascular. Si es gradual a incompleta sólo da Iugar a congestión venosa y trombosis consecutiva, pero si la oclusión es completa, sea esta gradual o súbita, trae consigo el infarto hemorrágico y la necrosis o gangrena de los tejidos. Vale la pena recordar que la trombosis puede continuar por el pediculo ovárico más allá del sitio de la torsión, hecho que hace necesario proceder a la ligadura del pedículo vascular en pleno tejido sano, muy por encima del sitio de la torsión, con lo cual se evitará la embolía post-operatoria. De la intensidad y duración de la torsión derivan lógicamente la agudeza de la sintomatología y así se explican los pequeños o soportables dolores de la mayoria de aquellas enfermitas con tumores ováricos a que aludíamos más arriba (casos 8, 12, 15 y 16), dolores que eran pasajeros y remitían periódicamente, durante uno o más meses antes de su hospitalización definitiva. Así también nos explicamos aquellos casos con grandes dolores abdominales, defensa muscular y vómitos (casos $8,11,13$ y 17), causados por súbita y fuerte torsión del pedículo ovárico, que clínicamente dieron el cuadro de abdomen agudo, no hieieron posible la palpación abdominal del tumor y sólo se sospechó una apendicitis aguda. En los otros casos con torsión (10, 12,14 y 16) la sintomatología fué menos ruidosa y también los hallazgos anátomopatológicos correspondientes en las respectivas piezas. De los 11 teratomas que comentamos, 7 sufrieron la torsión de su pedículo, lo que significó una incidencia del $64 \%$ más o menos en este grupo de tumores. De estos 7 casos. 3 eran quistes dermoides. Los 7 casos fueron operados de urgencia después de una enfermedad cuya evolución fluctuaba entre 1 y 7 días, pero la mayoría ( 5 casos) sólo alcanzó hasta los 3 días de enfermedad. El tiempo de su permanencia en el Hospital hasta el momento en aue fueron intervenidas osciló entre los 30 minutos y las 30 horas, operándose 5 de ellas hasta la cuarta hora de su ingreso. Abierto el peritoneo, en 6 se encontró líquido hemático en cantidad variable, a excepción de un caso ( $N^{\circ} 4$ ) en que sólo se advertía edema intenso de la serosa peritoneal. El pedículo se presentaba torcido en cuantía diversa, sobre- saliendo 3 casos $(8,12$ y 14$)$ en que un largo pedículo permitió la rotación del tumor en $180^{\circ}$ y 3 veces $360^{\circ}$ respectivamente.

Otra complicación que observamos en nuestras enfermitas fué la rotura espontánea de un tumor sólido maligno (caso 17). Este tumor, del tamaño de un puño de adulto $(10 \times 6 \mathrm{~cm}$.) mostraba múltiples rupturas que sangraban en regular cantidad y por algunas de las cuales asomaban mamelones carnosos de aspecto encefaloideo. El tumor adhería por una zona al recto, que aparecía infiltrado por tejido tumoral. En la cavidad abdominal se encontró gran cantidad de sangre líquida. Esta paciente, al ingresar llevaba 24 horas de dolores a la fosa iliaca derecha $y$ vómitos, síntomas que se acentuaron horas antes de su ingreso. Se operó de inmediato con el diagnóstico probable de apendicitis aguda. Según la literatura consultada, sabemos que se trata de una complicación rara, ya que las paredes de estos tumores son casi siempre bastante gruesas (Wharton). Quizás un traumatismo, cuyo antecedente no figuraba en la historia de esta enfermita, pudo haber jugado algún rol en su producción, tal como sucede en ciertas roturas de quistes dermoides.

Respecto de la histopatología, los Teratomas en general se caracterizan por contener tejidos procedentes de una o varias de las tres capas embrionarias (ecto, endo y mesodermo), tejidos que al combinarse pueden llegar a formar órganos 0 rudimentos de órganos, tales como piel, dientes, huesos, intestinos, tiroides, cabellos, bronquios, etc. La forma más simple de los Teratomas es el quiste dermoide, predominando en sus estructuras los derivados ectodérmicos, a base de tejidos adultos $\mathrm{y}$ especializados, de lo que resultan órganos mejor formados y más semejantes a los del individuo adulto. Sin embarga, con búsquedas prolijas es posible encontrar tejidos procedentes de las 3 capas germinativas. El componente entodérmico, antes raramente identificado en los quistes dermoides, se encontraria presente según algunos autores (Costin y Kennedy) en más del $50 \%$ de los casos. Con suma frecuencia los quistes dermoides contienen pelos y dientes, sumergidos en una atmósfera sebácea u oleosa. El pelo es muy largo, como el de la cabeza, o corto 
y fino como Ianugo, a rizado y crespo como el del pubis $y$ a menudo blanquecino en quistes de enfermas jóvenes. Según Ewing, en la mitad de los dermoides se encuentran piezas dentarias bien constituídas y que permiten su clasificación de igual manera que los dientes y molares normales. En algunos casos, éstos son numerosísimos $(100$ a 300 dientes según Schnabel y Plauquet). Por el estado de diferenciación de sus tejidos el quiste dermoide se desarrolla hasta cierto limite $y$ puede permanecer estacionario durante años. Las calcificaciones tisulares del quiste pueden hasta detener totalmente su crecimiento. Los teratomas y dermoides se asocian a menudo con quistes ováricos del tipo seroso, pseudomucoso o de revestimiento ciliado. Esta asociación es para Arnsperger del $14 \%$. Schottlaender los ha visto asociados también con quistes de cuerpo lúteo. En estos casos, la porción dermoide está relegada a un segmento de la pared quística a se proyecta como prominencia digitoide en un punto del auiste (protuberancia de Rokitansky). Pueden también estar uno al lado del otro, más o menos fusionados, $\mathrm{y}$ aún el dermoide asentar en un ovario $\mathrm{y}$ el cistadenoma en otro.

Macroscópicamente, el quiste dermoide es lobulado, cuyo tamaño varía desde un nódulo pequeño hasta un quiste voluminoso. Nace en el ovario mismo, al cual destruye o en el ligamento ancho a en el espacio retroperitoneal. En estas últimas ubicaciones ambos ovarios pueden estar normales y rastros de tejido ovárico supernumerario es posible encontrar en las paredes del quiste extraovárico. Generalmente el auiste dermoide es benigno. Su malignidad es rara, del $1.7 \%$ según Meyer y $3 \%$ según Blackwell, lo más común a partir de su revestimiento epiteljal plano pluriestratificado aue da lugar a un carcinoma epidermoideo. Se citan casos, sin embarco, de carcinoma mamario en un dermoide (Yamagiwa), o evolución sarcomatosa de estos quistes (Ludwig. Amann y Lorrain, etc.), adenoma de las glándulas sudoríparas del dermoide (Frjedlaender), etc. Para Hobne. la malionjzación es muy freruente en los dermoides bilaterales.

El Teratoma embrionario está constituido oor toda clase de tejidos que derivan de las tres hoias germinativas, muchos de ellos reconocibles al microscopio $y$ otros atípicos e irreconocibles. Moore dice que estos tumores muestran cualquier tejido del cuerpo humano, adulto o embrionario, con excepción de tejidos gonadales. Generalmente son tejidos embrionarios. Se diferencian de los dermoides por la ausencia de cavidades quísticas con pelos o sebo o extremidades definidas u órganos. Los tumores sólidos contienen a. veces numerosos y pequeños quistes que estudiados con atención permiten descubrir trozos de cartílago, dientes rudimentarios, dermoides minúsculos con finos pelos y abundantes células con estructura y morfología embrionarias. Los tejidos más comúnmente presentes son los que corresponden al tractus digestivo y respiratorio, elementos del sistema nervioso, hueso y cartílago. Musculatura lisa o esquelética se observan independientemente o en relación con órganos que las poseen. Llenando todos los huecos entre estos tejidos diferenciados, se encuentra abundante tejido fjbroso y adiposo, en los casos benignos. El Teratoma embrionario es por lo común de tamaño pequeño o moderado. En ocasiones su cápsula se rompe y el tumor invade los órganos vecinos. Su malignidad es muy frecuente, por lo que conviene practicar en cada uno un minuciosa estudio microscópico antes de catalogarlo como "benigno". La multiplicidad de tejidos que lo componen hace que cualquiera de ellos o varios a la vez, evolucionen hacia la malignidad, por lo cual son muy variados los tumores malignos resultantes e igualmente las estructuras que adoptan. Evoluciones peculiares, aunque raras, son el "struma" o "bocio" ovárico v el corio-epitelioma teratomatoso. Según Moore se encuentra el primero de estos en el 4-15\% de todos los teratomas. Emge afirma la existencia de casos auténticos cuya extirpación produjo la mejoría de un hipertiroidismo que afectaba al sujeto operado.

Las metástasis frecuentes son de los teratomas sólidos y embrionarios. Localmente. en el peritoneo, se producen diseminaciones por rotura del auiste o de la cápsula del tumor. Las metástasis por im-* plantación son de naturaleza muy variada y compleja. Por vía linfática son invadidos los ganglios retroperitoneales $v$ por vía sanguínea el pulmón, hígado, riñones, etc. Estas últimas metástasis son generalmente de tipo embrionario o sarcomatoso. 
Ewing cree que pueden desprenderse de Ios teratomas diversos tipos de células diferenciadas y desarrollar metástasis neoplásicas, pero que aún no está demostìado que las células indiferenciadas capaces de originar formaciones tridérmicas puedan desplazarse de los tumores ováricos.

Como conducta terapéutica se acepta que todos los tumares ováricos son potencialmente malignos y deben extirparse. Wharton aconseja abrir el quiste tan pronto se haya extirpado y si no tiene el aspecto macroscópico de un dermoide típico, proceder inmediatamente a la operación más radical. Salvo casos de tumores notoriamente malignos, este mismo autor es partidario de la laparotomía exploradora con el fin de comprobar fehacientemente la inutilidad de una operación. El uso de la Radioterapia pre o post-operatoria ha sido llevada a la práctica con resultados a veces halagadores. Parks, en 1945, publicó que el tratamiento con Rayos X había hecho operables 3 casos de Cistadenoma papilar, 2 de los cuales vivían en buena salud 8 y 12 años después respectivamente, aunque la tercera murió 5 años después de la operación.

La histogénesis de los Teratomas, aunque discutida, tiende a relacionarse con el óvulo en alguna de sus primeras fases de desarrollo, tal vez de blastómeros aisIados o supernumerarios. Las células multipotentes del blastómetro explican la producción de tejidos de las 3 capas germinativas y la capacidad para formar órganos. Otros hacen partir estos tumores de células germinativas gonadales o extragonadales que han sufrido el proceso de partenogénesis o de cuerpos polares fecundados.

En nuestra serie había 5 quistes dermoides ( $\operatorname{casos} 7,8,9,10$ y 11), todos unilaterales $y$ por rara coincidencia, todos del lado derecho. El mayor medía $9 \times 6 \mathrm{~cm}$. (caso 10) y el menor $1,5 \mathrm{~cm}$. (caso 7 ). Todos eran macizos con abundante sebo y pelos. Un caso ( $\mathrm{N}^{\circ} 9$ ) estaba implantado en el ligamento ancho. Tres de los cinco quistes presentaban su pedículo torcido - (casos 8,10 y 11), sufriendo las enfermitas un cuadro agudo abdominal que exigió la operación de urgencia. En ningún caso hubo rotura del quiste. EI tratamiento en 4 casos (el Nơ 7 fué hallazgo de autopsia) fué la salpingooforectomía, de la cual se recuperaron sin incidencias, salvo una de ellas (caso 11) que tuvo evisceración intestinal al $15^{\circ}$ día, siendo reoperada y dada de alta 10 días después.

El estudio microscópico de cada quiste dermoide demostró Ia existencia de tejidos ecto-mesodérmicos. No encontramos derivados entodérmicos. En uno (No 7) había escaso tejido ovárico en la pared quística. En los tres casos con torsión del pedículo, se observaron intensas lesiones de infarto hemorrágico.

Cuatro teratomas de nuestra serie (casos $12,13,14$ y 15 ) eran de tipo embrionario y quístico: 3 del lado izquierdo $y$ uno (caso 12) del derecho, todos de superficie externa lisa, sin adherencias de ninguna especie a los órganos vecinos. El más grande media $14 \times 10 \mathrm{~cm}$. y pesaba $420 \mathrm{gr}$. (caso 12) y el más pequeño era de $9 \times 6 \mathrm{~cm}$. (caso 15 ). En tres, el pedículo estaba torcido $(12,13$ y 14). Al abrirlos, se encontró en tres casos una gran cavidad quística (casos 13, 14 y 15) ocupada por líquido amarillento claro, más o menos teñido de rojo, en la cual había sendos nódulos murales, de $5 \mathrm{~cm}$. de diámetro en dos de ellos ( $N^{\circ} 13$ y 14) y de 2 $\mathrm{cm}$. de diámetro en el tercero (caso 15). Eri estos nódulos murales se encontró macroscópicamente elementos de quiste dermoide, tales como sebo y pelos en los tres casos y dientes y huesos en uno de los mismos (caso 14). El cuarto caso ( $N^{\circ} 12$ ) presentaba en su interior numerosas $y$ pequeñas cavidades. El estudio microscópico de estos 4 ejemplares, reveló en todos ellos abundantes tejidos provenientes de las 3 capas germinativas, pero de tipo adulto, predomínando los ecto-mesodérmicos: piel y sus derivados, cartílago y tejido fibro-adiposo en todos ellos, a lo cual se agregaba musculatura lisa en dos tumores (casos 13 y 15). Los tejidos entodérmicos estaban representados por glándulas o quistes revestidos por epitelio cúbico, cilíndrico o mucoso y en un caso (NQ 12) habían formaciones bronquiales. En los casos con torsión del pedículo había lesiones evidentes de infarto hemorrágico. No se encontró en ninguno de los 4 teratomas, nada sospechoso de evolución maligna. EI tratamiento quirúrgico en estos 4 casos consistió en la extirpación del tumor que incluía el ovario en 3 de ellos, pues en el otro (caso 13) el tumor se implantaba en el ligamento ancho $\mathrm{y}$ ambos anexos no revelaban alteraciones. En dos 
casos (No 12 y 15) hubo necesidad de extirpar también las trompas unidas al tumor. La convalescencia, en todas las enfermitas, se produjo sin accidentes.

Los dos últimos casos de Teratoma ( $\mathrm{N}^{\circ}$ 16 y 17) eran de tipo embrionario macizos, con francas estructuras histológicas malignas. Uno era del ovario izquierdo, de $15 \mathrm{~cm}$. de diámetro y $1 \mathrm{~kg}$. de peso, con su pedículo torcido, sin adherencias a los órganos vecinos, $y$ el otro, del ovario derecho, de $10 \times 6 \mathrm{~cm}$., roto en varias partes y adherido al recto, cuya pared estaba infiltrada y formaba cuerpo con la cara posterior de la masa tumoral. El primero, perteneciente a una niña de 10 años, se presentaba al corte formado por tejido blanquecino-amarillento, muy edematoso y sembrado de focos hemorrágicos y necróticos, hasta de $5 \mathrm{~cm}$. de diámetro. Microscópicamente, estaba constituido por diversos tejidos, algunos adultos y otros embrionarios, los primeros, derivados de las tres hojas germinativas y los embrionarios de calidad variada, en los cuales se apreciaban extensas zonas sarcomatosas y epiteliomatosas sembradas de numerosos focos hemorrágicos y necróticos. El diagnóstico histopatológico fué de Teratoma embrionario sólido maligno. La enferma fué dada de alta 15 días después de la operación y desgraciadamente no tuvimos más noticias de ella.

El otro caso ( $N^{\circ} 17$ ) procedente de una niña de 9 años, era del ovario derecho $y$ medía $10 \times 6 \mathrm{~cm}$. En varias zonas presentaba roturas como fisuras que daban salida a mamelones tumorales. Al corte era sólido, formado por un tejido blando, encefaloideo, Iiso, de aspecto necrótico. En un punto de su superficie había un nodulito sésil de $1 \mathrm{~cm}$. de diámetro, que al corte contenía varias pequeñas cavidades ocupadas por líquido espeso pardo-amarillento. El examen microscópico reveló un tejido francamente epitelial y de tipo maligno, con estructuras en que predominaban las formaciones glandulares. existiendo también zonas sólt́das y papilares. Las carioquinesis eran muy numerosas y los focos necróticos extensos. El nodulito de la superficie del tumor estaba constituido por tejidos adultos de las tres capas germinativas, pero parcialmente infiltrado por elementos del tumor maligno vecino. Controlada 2 meses después de la operación, presentaba mal estado general, gran masa abdominal del tamaño de una cabeza de feto en el flarico y fosa ilíaca izquierda, circulación venosa colateral y edema de los miembros inferiores. Todo hacía presagiar un final próximo. Por desgracia no volvió a control ní pudimos averiguar su suerte futura.

\section{Disgerminomas.}

Según Schneider y Vesell han sido descritos sobre 300 casos en la literatura hasta 1947. En general es un tumor raro, constituyendo menos del $3 \%$ de todos los tumores malignos del ovario (Wharton). De acuerdo con este último autor, se caracterizan clínicamente por presentarse en enfermas con desarrollo genital defectuoso, tal como hipogenitalismo y hermafroditismo. Se han descrito estos tumores asociados a otros: tumor de Brenner, fibroma, teratoma o corioma del ovario, etc. (Blanchard y Ewing).

Barzilai afirmó que más o menos un tercio de los casos publicados han ocurrido en niñas. Novak, desputés de una revisión de la literatura, concluyó que aparecen en edad temprana, pues la mayoría había alcanzado sólo a 6 años. En la serie de Costin y Kennedy de 22 tumores, que se refiere a tumores en la niñez, sólo había un caso de Disgerminoma, lo que representaba un 4,5\%. En realidad, muchos autores coinciden en afirmar que el reconocimiento de estos tumores es reciente, pues hasta no hace muchos años se incluían entre los sarcomas y carcinomas del ovario.

Fueron descritos por Chevasu en 1906 y Chenot en 1911, recomendando el primero la fijación precoz de estos tumores en líquido de Zenker para evitar la destrucción del frágil citoplasma celular. Estos mismos autores afirmaron que se originaban de células sexuales de tipo "indiferente", es decir, antes de su diferenciación en células sexuales machos o hembras, opinión manifestada antes de la aplicación de tests hormonales en el diagnóstico diferencial de tumores testiculares u ováricos. En 1931, Meyer introdujo la denominación de "Disgerminoma".

Su sintomatologia es la de cualquier tumor pélvico: tumefacción abdominal, dolor y síntomas de compresión de la vejiga y recto. Los más malignos pueden invadir los órganos vecinos y también dar 
metástasis a distancia. En estos casos la ascitis es frecuente, acompañándose de gran tumefacción abdominal.

Sus dimensiones son muy variables, pudiendo ocupar toda la pelvis y aún todo el abdomen. Su crecimiento suele ser rápido y alcanza considerables dimensiones antes de ser descubiertos. A menudo son superiores a los 2 kilos de peso. En el $33 \%$ según unos (Costin y Kennedy) y en el $\mathbf{5 0} \%$ según otros autores (Ewing) son bilaterales. Como un accidente raro se han descrito en la literatura dos casos de torsiones en Disgerminomas, por Narat y cols., a los cuales ellos agregan uno más.

Son encapsulados, de superficie externa lisa, duros o elásticos, a veces nodulares - groseramente lobulados por los tabiques conjuntivos que desde la cápsula irradian hacia la masa tumoral.

Microscôpicamente, se presentan constituídos por células grandes, redondas o poliédricas, de citoplasma clato que se tiñe escasamente y a menudo se destruye, quedando la célula reducida al núcleo, por lo que se le conoce también con el nombre de "Tumor de células claras". El núcleo se tin̄e fácilmente y es vesiculoso. Las células se disponen en grandes alvéolos o trabéculas separadas por bandas conjuntivo-fibrosas de espesor variable, infiltradas casi siempre por linfocitos. Los focos o zonas de necrosis tumoral no son raros, debido a su rápido crecimiento. Su estructura es idéntica a uno análogo que se presenta en los testículos. Seegar comparó el aspecto microscópico de estos tumores con un plato de caviar, comparación que puede resultar idónea, según Mandeville y cols., en zonas sin necrosis o hemorragias.

Su malignidad es muy discutida: algunos la estiman en alto grado y otros los consideran de malignidad variable. En el primer caso infiltran la cápsula y se adhieren a los órganos vecinos. Su propagación metastática la hacen típicamente por la vía linfática, razón por lo que Delbet le dió el nombre de "Carcinoma linfófilo": así afecta los ganglios regionales lumbo-aórticos en primer lugar, tomando enseguida en forma progresiva a los superiores $y$ posteriormente a los diferentes órganos (pulmones, riñones, etc.).

Su tratamiento es quirúrgico y la tendencia de la mayoría es practicar una ope- ración lo más radical posible, seguida de Radioterapia, recurso este últímo que a juicio de varios autores da buenos resultados, ya que se trata de tumores muy radiosensibles. La irradiación Roentgen también está indicada en casos con complicaciones glandulares y al menos produce alivio temporal (Wharton). Schiller dice que la cirugía puede curar del $70-90 \%$ de Ios casos en niñas.

Respecto de su histogénesis, Meyer los atribuye a células derivadas de la gónada primitiva en una fase muy precoz y antes que las células sexuales verdaderas les hayan comunicado los caracteres sexuales. Se funda esta hipótesis en la falta de propiedades sexuales $y$ de efectos hormónicos -estos tumores han sido encontrados hasta en mujeres embarazadas (Schneider y Vesel, Schoemaker y cols., etc.)y con frecuencia en tumores similares en el testículo.

En nuestra serie de 21 tumores del ovario, 2 corresponden a Disgerminomas, lo que significa una incidencia del $9,5 \%$. Uno de los casos ( $N$ \% 12) corresponde a necropsia y el otro a biopsia ( $N^{9} 19$ ).

En el $1^{\circ}$ (Caso 18). Niña de 9 meses, que ingresa al Hospital el 16 de Febrero de 1955, por presentar intensa tos desde hacía varios dias. Al examen se com. probó Coqueluche, Bronconeamonia y Tumor abdominal. Este último se palpaba por el abdomen, libre $y$ to doloroso. La enfermita se agravó progresivamente y falleció el 12 de Marzo de 1955.

En la Necropsia ( $\left.\mathrm{N}^{\circ} 193 / 55\right)$ se encontró el ovario izquierdo reemplazado por un tamor redondeado que media $9 \mathrm{~cm}$. de diámetro y pesaba $240 \mathrm{gt.}$ de color rosado-blanquecino con zonas rojizas y de consistencia blanda. Al corte presentaba color blanquecino-amarillento, sembrado por zonas rojizas y amarillentas $y$ en general muy edematoso. La superficie del corte era lísa y en las zonas edematosas aparecía algo lobulado.

Examen microscópico del tumor: La mayor parte del tumor se observó necrosado $y$ transformado en una masa eosinófila, en la que persistían siluetas de vasos sanguineos y de célnlas redondeadas. Cercana a la cápsula se encontró pequeñas zonas del tumor, cuyos elementos celulares se disponían difusamente, sin ordenación especial, surcadas a veces por alguna gruesa banda fibrosa. hialínica y limitadas externamente por cáp. sula fibrosa indemne. Las células tumorales etan grandes, redondeadas u ovoideas $y$ a veces ligeramente alargadas, de núcleo vesiculoso, más o menos claro, con granclaciones gruesas en número de 2 o 3 : su citoplasma era cosinófilo, regularmente abundante $y$ a veces escaso, reducido a delgado anillo perinusteat. Era 
casi frecuente encontrar núcleos que sobresalian por su tamaño e biperctomatismo, a veces doble o triples u observar parejas de células incompletamente divididas y una que otra carioquinesis. Numerosas células se encontraban en necrobiosis, permaneciendo bien conservadas aquellas que rodeaban los vasos sanguíneos. El estroma estaba formado por una que otra trabécula fibrosa, como ya se ha deserito. Y numerosos vasos sanguíneos con patedes propias. En zonas profundas de la cápsula se advertian vasos que contenian elementos tumorales. La cápsula no se apteciaba invadida en forma franca por el tumor.

No se enconeraron metastasis tumorales.

La causa inmediata de la muerte se debió a la Coqueluche.

En el $2^{\circ}$ (Caso 19). Niña de 10 años. Consultó el 21 de Diciembre de 1951 , porque el mes anterior presentó una menarquia, A los 25 dias nuevamente tuvo pérdida de sangrte.

Examen fisico: Niña en buen estado general. algo delgada, consciente. Piel con turgor y elasticidad normal. Cabeza: nada especial. Cuello: no se palpan ganglios. Tórax: en la pared anterior se observa red venosa aumentada. Pulmones: matidez en ambas bases. Corazón: ruidos cardíacos normales. Abdomen: mo. vifidad respitatoria disminuida. Abombamiento supraumbilicai. Se palpó an tumor que ocupaba el epigastrio, parte de ambos hipocondrios y región umbilical. Sa consistencia era dura, bien limitada. no adberido a la piel. La piel que lo recubría no presentaba alteraciones. Se palparon en la región inguinal mitropoliadenaia. Percusión: matidez. Auscaltación negativa. Extremidades: nada especial.

Impresión diagnóstica: Tumor abdominal.

Operación: Laparotomía media infeambilical.

Abierto el peritoneo se apreció la existencia de un tumor sólido cuya implantación correspondia al anexo izquierdo. Líquido peritoneal en los fondos de saco. Salpingo-ooforectomía izquierda. Peritonización de los muñones. Saturas por planos. Montura.

Examen anátomo-patológico: Biopsia 364/51.

Tumor que pesó $1.410 \mathrm{gr}$., de forma ovoidea $\mathrm{y}$ de $15 \mathrm{~cm}$. de diámetro. Todo el tumor se encontraba rodeado de una delgada capa fibrosa $y$ en uno de sus bordes se observaba nna trompa y al lado de ella un órgano del tamaño de una aviltana que parecia corresponder a na ovario. Al corte el tumor se presentaba de color sosado-blanquecino. de superficie lisa, con numerosos focos edematosos y algunos hemortágicos.

Microscópicamente el tumor se encontraba formado por células redondeadas muy iguales cntre si, de citoplasma may frágil que no tomaba los colorantes. apareciendo claro $y$ a veces ligeramente cosinófilo: núcleos con finos gránalos cromáticos. Carioqninesis numerosas. En escasos puntos se observaban célnlas de tamaño y forma variable, de zúcleo hipercromático $y$ a veces gigantes. Las células se conglomeraban en nódulos sólidos de tamaño diferente, limitadas por delgadas trabéculas conjuntivas, pobres en colágeno. En algunas zonas el estroma era abundante. fibroso e infiltrado por pequeños focos linfocitatios. La cápsala que limitaba el tamor se observaba invadida en numerosos puntos por nodrlitos tumorales, alcanzando en ocasiones hasta la superficie de ella.

Trompa: Sin alteraciones microssópicas.

El órgano con aspecto macroscópico de ovario, vecino a la trompa, estaba formado por tejido célnlo. adiposo y gruesos vasos sanguineos, sin que se encontrara en ê], tejïdo ovárico (Parametrío).

Controlada hasta 4 años después de la operación. estaba en buenas condiciones. Se hizo radioterapia postoperatoria.

Como puede observarse, ambos casos eran unilaterales y el primero de 9 meses de edad, tal vez el más joven que figura en la literatura. En los dos casos, el tumor estaba libre y eran francamente malignos desde el punto de vista histopatológico, con vasos sanguíneos que contenían elementos tumorales en el primero y con invasión de la cápsula en el segundo, pero en ninguno de ellos se presentaron metástasis, aun cuando en el segundo caso transcurrieron 4 años después de la operación.

\section{Carcinomas sólidos.}

Este grupo de tumores malignos del ovario, se denomina también "Carcinomas no clasificados" o "Carcinomas indiferenciados". Se trata de tumores primitivos del ovario que proceden directamente de este órgano y que adoptan estructuras variadas o mixtas: las más comunes son las formas sólidas, papilares o adenocarcinomatosas. Iros carcinomas papilares pueden derivar de un Cistadenoma papilar, es decir, se trataría de un Cistadenocarcinoma papilar, en cuyo caso el quiste no se desarrolló o fué totalmente ocupado por las células tumorales. Como es obvio. están excluidos los tumores malignos teratomatosos y aquellos que se originan a partir de restos embrionarios: tumores de células granulosas, disgerminomas y arrenoblastomas.

Su frecuencia, según Stout, es del $\mathbf{5} \%$ de todos los tumores malignos femeninos. Según Moore son más comunes entre la quinta y sexta década de la vida, incidiendo el $40 \%$ de ellos en mujeres menopáusicas. Los carcinomas sólidos se des- 
arrollan en o sobre la superficie del ovario, afectando pronto todo el órgano. En un $40 \%$ de los casos son bilaterales. Aparecen como una masa sólida, firme, lobulada o lisa, de tamaño variable. Rápidamente invaden los tejidos vecinos, los ligamentos anchos y el peritoneo. En la cavidad peritoneal se acumula abundante líquido. Las metástasis son frecuentes, en especial en los pulmones. Macroscópicamente, el tejido tumoral es blanco-grisáceo, con numerosas y pequeñas zonas amarillentas de necrosis o hemorragias. No son raras las formaciones quísticas. El aspecto microscópico corresponde a un tumor compuesto por abundante estroma fibroso que incluye células epiteliales atípicas que se disponen en trabéculas y nó dulos. El tipo más común es el carcinoma sólido, pero no son raros el adenocarcinoma y el carcinoma mucoso. Es propio del carcinoma ovárico, la intensa variabilidad de la morfología celular'y la anaplasia tan notable de sus elementos. Los de estructura papilar son rápidamente invasores, dando lugar en el peritoneo a extensas implantaciones $\mathrm{y}$ ascitis.

Todos están acordes en que los carcinomas sólidos son los más malignos. Según Jacob su evolución no excede de año y medio y la sobrevida post-operatoria sobre 5 años es menos del $10 \%$ de los casos. Los quísticos en cambio tienen un pronóstico mucho mejor, alcanzando hasha el $35 \%$ de sobrevida post-operatoria sobre $\mathbf{5}$ años.

En nuestra casuística tenemos 2 casos de carcinoma sólido $(9,5 \%)$.

El $1^{\circ}$ (caso 20) se trataba de una niña de 10 años que ingresó a la Posta Infantil por presentar desde hacia 3 días fuertes dolores abdominales. Se diagnosticó peritonitis generalizada de origen apendicular y se operó de inmediato. En la cavidad peritoneal habia líquido hemático y coágulos $y$ un tumor del ovario izquierdo del tamano de una cabeza de niño, torcido, que al tratar de exticparlo se rompió. El estudio atátomo-patológico reveló un cáncer sólido $y$ en partes papilar, con extensas zonas de necrosis y hemorragia. El carcinoma sólido presentaba gran variabilidad en el tamaño $y$ forma de sus elementos y eta excepcionalmente rico en carioguinesis. La cápsola del tumor se presentaba invadida, basta la snpetficie externa de ella, por nódulos tumorales $y$ formdciones papilares. A esta niña se le bicieron posteriormente 20 sesiones de radioterapia. Su estado general fué decayendo rápidamente y falleció con gran bepato- megalia (presumiblemente por metástasis tumoral) enflaquecimiznto y edemas, 3 meses después de operada.

E1 $2^{\circ}$ (caso 21) era una niña de 13 años que acudió a la Posta Infantil por traumatismo abdominal que le ocasionó agudo dolor al abdomen, pézdida del conocimiento, vómitos y gran malestar general. Un mes antes se habia palpado en tumor abdo. minal. En el examen se apreció intensa ascitis y gtan tumor abdominal doloroso, baciéndose diagnóstico de quiste del ovatio derecho roto y se intervino de $\mathrm{ur}^{*}$ gencia. En la cavidad peritoneal se encontró abundante líquido tosado (6 litros más o menos) y gran tumor det ovario derecho roto, de superficie ittegular por la presencia de numerosos mamelones carnosos con aspecro de tamor maligno. Se practicó salpingoofoxectomia derecha. Se observaron además numerosos ganglios mesentéricos infartados $y \mathrm{l}_{2}$ serosa intestinal sembrada de placas gris-amarillentas de aspecto tumoral. El examer anitorro-patológico del tamor (de $13 \mathrm{~cm}$. de diámetro) reveló un adenocarcinoma parcialmente milcoso, cuyos elementos eran muy variables. may cromatínicos. con carioquinesis en regular cantidad y numerosos focos necróticos. El tejido tamoral infiltraba extensamente la cápsula fibrosa, apareciendo al exterior en forma de nódalos hasta de $1 \mathrm{~cm}$. de diámetro. Se envió también a Radioterapia. practicándosele hasta 4 sesiones y 2 paracentesis que evacuaron más o menos 5 litros cada una. La enferma, ya caquéctica, con gran ascitis, falleció dias después, a los 2 meses de haber sido intervenida.

En ambos casos, el tumor evolucionó silenciosamente y sólo un accidente reveló su presencia: la torsión en el $1^{\circ}$ y el traumatismo abdominal en el $2^{\circ}$. Si bien en este último caso, se habría palpado el tumor un mes antes, éste era indoloro y no preocupó a la portadora. En ambos, la malignidad histopatológica fué evidente y ya al tiempo de la operación había metástasis que en el $2^{\circ}$ caso fueron vistas por eI cirujano. Finalmente, el deceso ocurrió en ambas, a los 3 meses de haber sido detectados los respectivos carcinomas.

\section{COMENTARIO GENERAL}

La frecuencia de Tumores del Ovario en nuestro material de biopsias de niñas, 16 entre 1.174, fué de 1,03\%. En necropsias, 5 casos entre 3.551 autopsias de niñas, sólo alcanzó al $0,1 \%$. Aunque la revision de nuestra casuistica abarca desde 1943 hasta la fecha, observamos que en el material de biopsias, el hallazgo de estos tumores es mucho más frecuente en el último lustro ( 9 casos, $56,25 \%$ ) que en 
TUMORES DEL OVARIO. - Dr. Julio Espinoza y fol.

CUADRO NO 4

TUMORES DEL OVARIO EN LA INFANCIA

(21) casos)

ALGUNAS CARACTERISTICAS ANATOMO.CLINICAS

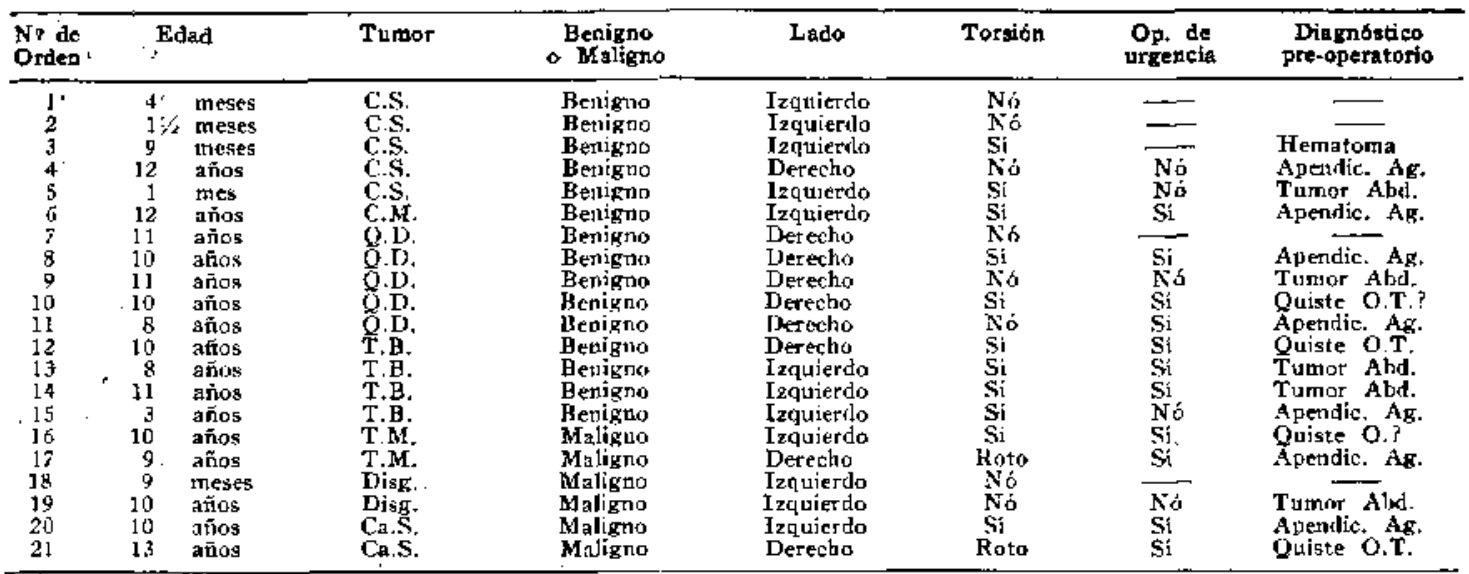

Abreviacianes: C.S. $=$ Cistadetioma Sernsa. C.M. = Cistarlenoma mucaso. Q.D. = Quiste alermoide. T.B. = Teratoma Benigno. T.M. = Teratoma maligno, Disg. = Disgerninoma. Ca.S. = Circinoma sólido. Quiste O.T. $=$ Quiste Ovario. Torcido.

los 9 años anteriores ( 7 casos). Tal vez podamos explicarlo por la mayor afluencia de niños con cuadros agudos del abdomen, sindrome bajo el cual se presentan muchos de estos casos, que han sido atendidos desde que funciona la Posta Infantil de nuestro Hospital.

Respecto de la edad de los individuos en que se presentaron los tumores analizados (Cuadro $\mathrm{N}^{\circ} 4$ ), la gran mayoría, 15 casos $(70 \%)$ estaba sobre los 8 años, 5 casos (24\%) entre 1 y y 3 meses y 1 caso a los 3 años. Según lo expuesto, observamos que en el grupo de los lactantes, sólo uno de los tumores era maligno (caso 18), correspondiente a un Disgerminoma, mientras que el resto de los tumores malignos ( 5 casos) estaban ubicados en niñas sobre 8 años de edad.

Del' total de Tumores, 15, o sea el $71 \%$, correspondieron a tumores benignos $\mathrm{y}$ el resto a malignos $(29 \%)$.

Según el lado de ubicación del tumor, hubo leve predominancia del izquierdo $(57 \%)$ sobre el derecho. Llamó la atención que todos los Teratomas benignos se encontraron en el ovario derecho y casi todos los Quistes Dermoides en el izquiterdo.

Con suma frecuencia encontramos la torsión del pedículo tumoral en nuestra casuística, ya que 11 casos la presentaron, incidencia equivalente al $\mathbf{5 2} \%$ más o menos, accidente relativamente más frecuen- te en el grupo de los Teratomas: 7 veces en 11 casos $(64 \%)$. Otro accidente menos frecuente por cierto, es la rotura del tumor en dos casos (17 y 21), que, al igual que los anteriores, promovieron graves síntomas abdominales agudos y que obligaron la intervención quirúrgica de urgencia. Debemos agregar que, pese a lo urgente de la operación, que no permitió muchas veces una adecuada preparación de las enfermitas, todas vellas, a excepción de una (caso 11), tuvieron un post-operatorio sin incidentes respecto del acto quirúrgico mismo, pues sólo fallecieron con posterioridad aquellas afectas de graves díseminaciones neoplásicas (caso 20 y 21 ).

Los síntomas principales que pudimos observar en las enfermitas que comentamos (eliminados los 5 casos de necropsia). fueron el dolor y los vómitos en 14 de elias $(87,5 \%)$, en 6 de las cuales coexistían ambos sintomas y en 8 solamente estaba presente el dolor. En las 2 restantes, observamos metrorragia en una y constipación en otra.

El examen abdominal reveló dolor en todas ellas ( $100 \%$ ) acompañado de tumor en 9 casos (56\%) y de defensa muscular en $6(37,5 \%)$.

El diagnóstico pre-operatorio en estos mismos 16 casos; fué de Apendicitis aguda en 7 oportunidades $(44 \%)$, tumor abdominal en $5 \mathrm{y}$ quiste del ovario en 4 ocasiones, o sea, en 9 casos $(56 \%)$ se inter- 
vino para extraer un tumor del abdomen. El diagnóstico de apendicitis aguda, ya lo dijimos, fué el resultado lógico de la sintomatología abdominal de la torsión aguda o subaguda del pedículo tumoral. Creemos que el examen bimanual rectoabdominal y el mayor uso de los Rayos X, podría ser de gran ayuda en estos casos.

Se intervino de urgencia en 11 de estos 16 casos, o sea en el $69 \%$, correspondiendo los 11 operados a tumores con su pedículo torcido ( 9 casos) o con rupturas múltiples de la masa tumoral (casos 17 y 21). En un caso en que se encontró pedículo toreido ( $N^{\circ} 5$ ), no se intervino de urgencia por falta de síntomas que permitieran su diagnóstico. Corresponde este caso, a una enfermita de sólo 1 mes de edad, la más joven de la serie, hecho al cual atribuímos la carencia de fenómenos sintomáticos abdominales agudos.

El tratamiento efectuado en estas enfermas fué eminentemente conservador, respetando la trompa cuando fué posible ( 3 casos). En un caso (No 13), el tumor era del ligamento ancho y con largo pedículo, lo que permitió una quistectomia sin comprometer el anexo. En los casos macroscópicamente malignos ( $\mathrm{N}$ \% $17 \mathrm{y}$ 21), se observó durante la intervención, no sólo los mamelones neoplásicos que afloraban por las roturas de la masa tumoral, sino además implantaciones múltiples en el peritoneo, lo cual hacía innecesaria una operación más radical a nivel de los genitales. Efectivamente, el último caso falleció a los 3 . meses de operado y el primero tuvo a los 2 meses grandes masas abdominales, seguramente metástasis $y$ estado caquéctico, preludio del desenlace próximo. De los otros 3 casos de tumores microscópicamente malignos correspondientes a biopsias $(16,19$ y 20$)$, el $1^{\circ}$ se dió de alta a los 15 días de operada y no se tuvo más noticias de ella, el $2^{\circ}$, tratado con Radioterapia después de la operación, estaba bien hasta 4 años después de operada y el últímo falleció a los 3 meses de operada, por metástasis hepáticas, pese a 20 sesiones de Radioterapia a que fué sometida después de la operación. En resumen, de 5 enfermitas afectas de tumores: malignos, 3 sucumbieron a la enfermedad, correspondiendo 2 de éstas a Carcinomas sólidos. La sintomatología de estos 5 casos no fué diferente a la de aquellos en que se encontraron tumores benignos.

\section{RESUMEN}

Se analizan 21 casos de tumores del ovario, en niñas cuyas edades oscilan desde 1 mes a 13 años; 16 casos fueron diagnosticados por biopsia y 5 por necropsia.

De los 21 casos, 6 fueron cistadenomas $(28,5 \%), 11$ teratomas (incluyendo 5 quistes dermoides, $52,0 \%$ ), 2 disgerminomas $(9,5 \%)$ y 2 carcinomas sólidos $(9,5 \%)$.

15 de estos tumores $(71 \%)$ se encontraron en niñas cuyas edades estaban por encima de los 8 años y 5 en lactantes entre 1 y 3 meses de edad. 15 casos ( $71 \%$ ) fueron benignos y 6 casos (29\%) malignos. 12 casos estaban localizados en el lado izquierdo $(57 \%)$ y 9 en el lado derecho $(43 \%)$. No se encontró ningún caso bilateral.

La torsión del pediculo tumoral se encontró en 11 casos (52\%), siendo especialmente frecuente en el grupo de los teratomas: 7 de 11 casos $(64 \%)$. En 13 de los casos se observaron complicaciones derivadas del mismo tumor (11 torciones y 2 rupturas), con una incidencia de $62 \%$.

Los síntomas principales en 16 de las pacientes fueron el dolor abdominal y los vómitos que se observaron en 14 de ellas $(87,5 \%)$. El examen clínico del abdomen demostró dolor en todos ellos $(\mathbf{1 0 0 \%})$, con presencia de tumor en 9 casos (56\%) y defensa muscular en 6 casos $(37,5 \%)$.

El diagnóstico pre-operatorio fué de apendicitis aguda en 7 casos $(44 \%)$, tumor abdominal en $5(31 \%)$ y qujste del ovario en los 4 restantes $(25 \%)$.

En 11 de 16 casos fué necesario practicar un tratamiento de urgencia $(69 \%)$, en 9 por torsiôn del pedículo y en 2 por ruptura del tumor. De 5 pacientes con tumores malignos, 2 fallecieron 90 dias después de ser operados y en un tercer caso, probablemente sucedió lo mismo.

Ambos casos de disgerminoma mostraron un cuadro histológico de aspecto maligno; una de ellas recibió radioterapia post-operatoria y 4 años después estaba bien. Otro caso, comprobado por necropsia, que no presentaba metástasis macroscópicas, tenía 9 meses y es el caso de menor edad encontrado en la literatura consultada.

Los 2 casos de carcinoma sólido, hicieron una evolución asintomática $\mathrm{y}$ fueron descubiertos accidentalmente (torsión y ruptura, respectivamente) y ambos mu- 
rieron a consecuencia de metástasis $3 \mathrm{me}$ ses después de la operación.

De los 6 casos de tumores malignos, 5 fueron en niñas de más de 8 años de edad y sólo 1 en una lactante.

Finalmente, se analiza la etiología, patogenia, clínica y anatomía de cada grupo de tumores.

\section{SUMMARY}

\section{Ovary Tumons IN Infancy and Child- HOOD.}

21 cases of ovary tumors were analyzed, which age ranged between one month and 13 years; 16 cases were biopsies and 5 necropsies.

Of the 21 cases, 6 were cystadenomas $(28.5 \%), 11$ teratomas (including 5 dermoid cysts, $52 \%$ ), 2 dysgerminomas $(9.5 \%)$ and 2 solid carcinomas $(9.5 \%)$.

15 of these tumors $(71 \%)$ were found in individuals above 8 years old and 5 within the first three months. 15 cases ( $71 \%$ ) were benignant and 6 cases $(29 \%)$ malignant tumors. 12 were located in the left side $(57 \%)$ and 9 in the right $(43 \%)$. No bilateral tumors were found.

Torsion of tumoral pedicle was found en 11 casos $(52 \%)$, being it specially frequent in the teratoma group; 7 of 11 $(64 \%), 13$ had complications derived from tumor itself (11 torsions and 2 ruptures) meaning an incidence of $62 \%$.

The main symptoms in 16 patients, were abdominal pain and vomitings, observed in 14 of them (87.5\%). Abdominal examination showed pain in all of them $(100 \%)$, with tumor in 9 cases $(56 \%)$ and muscular defense in $6(37.5 \%)$.

The pre-operation diagnosis was acute apendicitis in 7 cases (44\%), abdominal tumor in $5(31 \%)$ and ovarian cystic in the other $4(25 \%)$.

11 out of 16 were treated urgently $(69 \%): 9$ for torsion of pedicle and 2 for the tumor rupture. From 5 patients with malignant tumors, 2 died 90 days after operation and a third one did also very probably in similar condition.

Both Dysgerminomas showed malignant histological picture: one of them was treated with post-operation radiotherapy and 4 years afterwards she was well. Another one, a necropsy case, without macroscopical metastasis, was 9 months old and it was the younger specimen found in consulted literature.

Both Solid Carcinoma, did develop inadvertently and were discover for an accident (torsion and rupture respectively) and both died with metastasis 3 months after operation.

Out of 6 cases of malignant tumors, 5 were in girls above 8 years old and only 1 was an infant.

Finally, etiology, pathogeny, clinic and anatomy of each group of tumors were analyzed.

\section{BIBLIOGRAFÍ}

1.-ARJZTIA, R. - "Tumores de! Ovario en ls Infaucia". Tesis. Universidad de Chile. 1949.

2 -BLANCHARD, O. - "Disgerminoma del Ovario". Arch. de 1 a Soc. Cir. de Chile. 3:4, $195 \mathrm{f}$.

3. COSTIN, M.E y KENNEDY, R. - "Ovarian tumors in infants and in children". Ain. J. Dis. Child. 76:2, 1948 .

A. DARGEON, H. W. - "Ovarian tumors in childhood". Pediatrics. $3: 6,1949$.

5.-DOUGLAS, G. F.; CAPERS, G. DOUGLAS, G. F. Jr. "Tumors of the Ovaty". J. of Int. Coll. of Surg. 16:5, 1951 .

6.-EWING, J. - "Oncologia". Salvat Editores S. A., Barcelona, B. Aires, 1948.

7.-GARRET. S. S. "Teratona of the Ovary in at Infant wenty months of age". Ath. J. Dis, Child. $79: 2$, 1950 .

8.-GRAVES, G. I.; Ic. ILVOY, D. B. y HUDSON, G. W. "Ovarian cyst in a prentature infant". Am. J. Dis, Child, 81:2, $19 \$ 1$.

9.-LTSA, J R.; HINTON, J. W.; WIMPHEIDER, S. $\checkmark$ GIOIA, J. W. - Malignant ovarian teratomas in the first two recades of life". Am. J. of Surg. $81: 4$, 1951 .

10.-MA.NDEVILLE, F. B.; SAHYOUN, P. F. y SUTTON, L. E. - "Disgerminoma of the avary in a four-year old girl with metastaseg clinically simulating Wilms tumor atd adrenal neuroblastoma". J. Pediat. $34: 1,3949$.

11.-MOOR, R. A. - "A Textbook of Pathologg". W. B Saunders Co., Pbiladelobia and London, 1944.

12,-NARAT, I. K.: RAIDER, J. H. y CARTOU, A. K. "Torsion of Dysgerminoma of the Opary in a Child". Surgery. 30:5, 1951 .

13.- SMITH, $R$. C. - "Simple ararian cyst in a newborn iniant". Pediatrics, 1t:3, 1954.

14 -WHARTON, L. R - "Gibecología". Editorial Interśmericana S. A., Jéxico, 1950. 